\title{
Design of CMOS UWB Noise Amplifier with Noise Canceling Technology
}

\author{
Ya Gao ${ }^{1}$, Ningzhang Wang ${ }^{1}$, Yan Zhao ${ }^{2}$ \\ ${ }^{1}$ Academy of Computer and Electronics \& Information, Guangxi University, Nanning 53004, China \\ ${ }^{2}$ Beijing Research Institute of Telemetry, Beijing, 100076, China \\ saraggccxx@126.com, zhaoyan0618@126.com
}

\begin{abstract}
A TSMC $0.18 \mu \mathrm{m}$ RF CMOS low-noise amplifier (LNA) for $3 \mathrm{GHz}-5 \mathrm{GHz}$ ultra-wideband (UWB) applications is presented. The designed LNA employs single-ended to differential conversion and is successfully implemented using the noise-canceling technique. This paper introduces common-gate stage performance and noise figure(NF)optimization. Simulation results show that the proposed circuit network achieves a voltage gain of $17.34 \mathrm{~dB}-19.6 \mathrm{~dB}$ and noise figure of $2.02 \mathrm{~dB}-2.67 \mathrm{~dB}$ over the band of interest, consuming $12.5 \mathrm{~mW}$ from a $1.8 \mathrm{~V}$ power supply voltage.

Index Terms - CMOS, UWB, low-noise amplifiers
\end{abstract}

\section{Introduction}

As a new technology of high speed wireless short distancecommunication, ultra-wideband technology has been widely investigatedbecause of the advantages of low powerco nsumption, high rate, and anti-interference-ability.

This paper proposes a CMOS Ultra-wideband low noise amplifier with noise canceling technology over $3 \mathrm{GHz}-5 \mathrm{GHz}$.

Some excellent wideband input impedance matching solutions are proposed in[1]:1) The distributed amplifier. It achieves good wideband matching, but occupied area make it difficult to meet the requirements of the UWB LNA; 2) The resistive shunt feedback amplifier. It provides wideband input matching by local feedback, but it consumes large power dissipation; 3) The bandpass filter. Wideband input matching, low noise, flat gain and low power consumption are provided easily. However, requirements of a lot of high-Q inductors at the input is difficult to be realized in a small area; 4)The common gate(CS) stage[5]. It gains good impedance matching due to the inherently transconductance of CMOS, while causing high NF problems.

In this paper, the performance of noise is optimized using TSMC $0.18 \mu \mathrm{m}$ technology based on a single-end to differential balun circuit structure. The simulated noise figure of the proposed circuit is less than $2.7 \mathrm{~dB}$ and gain is greater than $17 \mathrm{~dB}$ over $3 \mathrm{GHz}-5 \mathrm{GHz}$ band.

\section{Input Impedance-Matching Design}

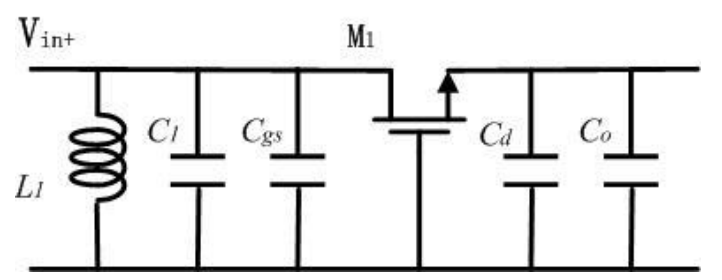

Fig.1. Common Gate M1 Small Signal Equivalent Circuit Diagram
Input matching circuit of the UWB LNA is composed of common gate transistor (CG) M1, L1, C1, as shown in figure 1, where $C_{g s}$ is the gate source capacitance, $C_{d}$ is the drain capacitance. The main noise contribution of CMOS amplifier is channel noise, given as

$$
F=1+\frac{\gamma}{\alpha}+\frac{4 R_{S}}{R_{L}} .
$$

where $R_{L}$ is the load resistance of the CG transistor, $R_{S}$ is the source impedance. Assuming $\gamma / \alpha=1.33$, the noise figure NF is about $4 \mathrm{~dB}$. In order to optimize system noise figure, we design a novel structure of the UWB LNA using the noise cancelling technology in this paper.

The input impedance of the UWB LNA is given as [5]:

$$
Z_{i n} \approx 1 / g_{m}
$$

Matching input impedance to $50 \Omega$, the transconductance of the CG transistor can be obtained as $g_{m}=20 \mathrm{mS}$.

\section{Noise-cancelling Technology}

The principle of noise canceling technology is described below. As shown in figure 2, the signal current $i_{\text {in }}$ flowing

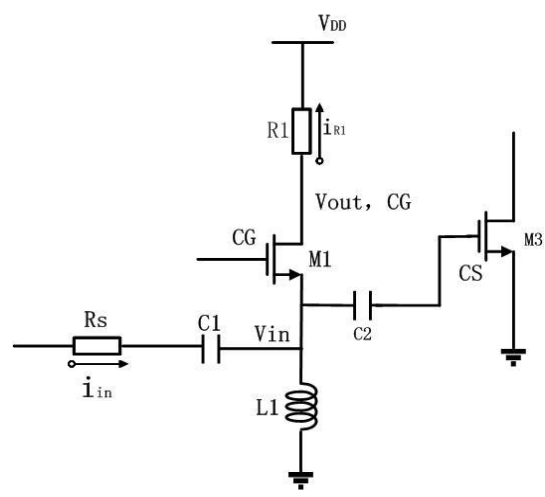

Fig. 2. Theory of a CG-Stage

through the load resistance has to be equal to the signal current flowing at the input $i_{R 1}$, that is, $i_{i n}=i_{R 1}$. The current can be expressed as the ratio of the input voltage and input impedance[2]: 


$$
i_{i n}=\frac{V_{i n}}{R_{i n, C G}}=\frac{V_{i n}}{R_{s}} .
$$

where, input impedance of the CG stage matches source impedance $R_{i n, C G}=R_{S}=50 \Omega$.

$$
i_{R 1}=\frac{V_{\text {out }, C G}}{R_{1}}=\frac{A_{C G} V_{i n}}{R_{1}} .
$$

$V_{\text {out, CG }}$ is the output voltage of the M1, whose gain $A_{C G}$ is written as $A_{C G}=\frac{V_{\text {out, CG }}}{V_{\text {in }}}$, and incorporating (3), (4), we have

$$
A_{C G}=\frac{R_{1}}{R_{s}}
$$

\subsection{Balun-LNA Topology}

Three different designs of the Balun circuit are analyzed, as follows:

1) The designed transconductance of the $\mathrm{CS}$ and the $\mathrm{CG}$ transistors is equal, $g_{m(C S)}=g_{m(C G)}$.

2) The designed transconductance of the CS stage is to be about times higher than the CG transconductance, $g_{m(C S)}=n g_{m(C G)}$.

3) The designed transconductance of the CS stage is to be about times higher than transconductance of the CG stage,

$$
g_{m(C S)}=n g_{m(C G)} \text {. }
$$

In order to achieve a smaller noise figure, the width of the CS is set as 4 times as large as the CG for better choices. The transconductance of the common gate is $g_{m}$. According to the selection of the width, the transconductance of the CS is $4 g_{m}$. The amplification factor of common-gate can be expressed as: $A_{C G}=g_{m} R_{1}$.

To create circuit balance, it can be expressed as:

$$
A_{C G}=-A_{C S}=4 g_{m} \cdot R_{1} / 4 \text {. }
$$

According to (6), $R_{1}$ is setting $1200 \Omega$ and $R_{2}$ is $300 \Omega$. The output voltage $V_{\text {out, diff }}$ of the differential circuit is expressed as follows:

$$
V_{\text {out diff }}=A_{C G}-A_{C S}=2 A_{C G} \text {. }
$$

According to equation (7), the signal is strengthened through the differential circuit output.

As shown in figure 3 and TABLE I, when the signal is amplified in phase through the M1, the signal at node $\mathrm{X}$ has the same phase with $\mathrm{Y}$ node. When the signal is amplified in anti-phase through the $M 3$, the signal at node $X$ has the opposite phase with $\mathrm{Z}$ node. Since the $\mathrm{Y}$ node and $\mathrm{Z}$ node has the same amplification factor in opposite phase, therefore the signal are enhanced through the differential output side.

As shown in figure 3 to TABLE I, the phase of current noise at node $\mathrm{X}$ is opposite to node $\mathrm{Y}$, and $\mathrm{X}$ node is opposite to $\mathrm{Z}$ node, , the current noise signal at node $\mathrm{Y}$ and at node $\mathrm{Z}$ are in same phase. The common mode noise of two signals

\begin{tabular}{|c|c|c|}
\hline & Signal & Noise \\
\hline $\mathrm{X}$ node & & \\
\hline Y node & & \\
\hline$Z$ node & & \\
\hline
\end{tabular}
with the same amplitude can be eliminated through the differential circuit.

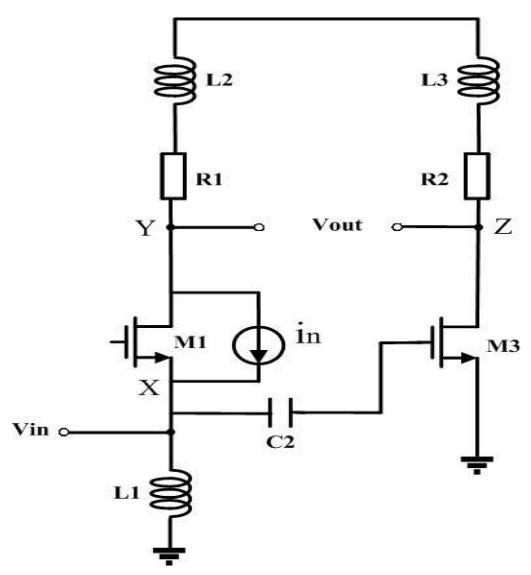

Fig.3. Circuit of Single-ended to Differential Conversion

TABLE I UWB LNA Phase Analysis

\section{Simulated Result Analysis}

As shown in figure 4, whole circuit of the UWB LNA using a noise cancellation technology. Figure 5 to 8 show Simulated S parameters and NF.

From Fig.5-Fig.6, input reflection coefficient S11 is below $-10 \mathrm{~dB}$, and output reflection coefficient S22 is less than $-11 \mathrm{~dB}$. It can be seen that good performance of input matching and output matching. The reverse isolation S12 is less than $-60 \mathrm{~dB}$ over the entire frequency, which indicates that reverse isolation of the circuit is better.

From the Fig.7, the circuit gain S21 larger than $17 \mathrm{~dB}$ in the frequency range of $3 \mathrm{GHz}-5 \mathrm{GHz}$ is obtained. The quality factor Q is greater than 8 all over the whole frequency range. $\mathrm{L} 1$ and $\mathrm{C} 1$ are resonated at low frequency point, effectively improving the input matching characteristics and low-frequency gain.

From the Fig.8, The noise figure is below $2.7 \mathrm{~dB}$ and the minimum NF of $1.88 \mathrm{~dB}$ occurs at $3 \mathrm{GHz}$ and $2.3 \mathrm{~dB}$ occurs at 
$5 \mathrm{GHz}$. It is shown that the frequency is higher, noise figure is worse, because characteristic of the CS transistor causes deterioration of noise.

The UWB LNA consumes $12.5 \mathrm{~mW}$ with a $1.8 \mathrm{~V}$ supply voltage. Table I summarizes the performance of the proposed UWB LNA and makes a comparison of the circuit with the simulation result of the recently published LNAs. The designed circuit using the noise canceling technology has a better simulation results in the gain、 noise figure insertion loss and power compared with some previous published works.

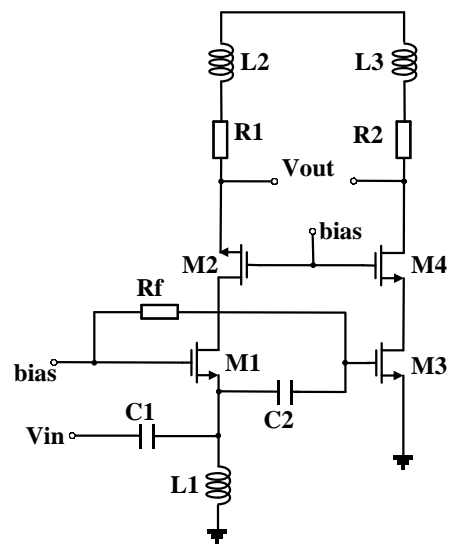

Fig.4. Whole Circuit of the UWB LNA Using a Noise Cancellation Technology

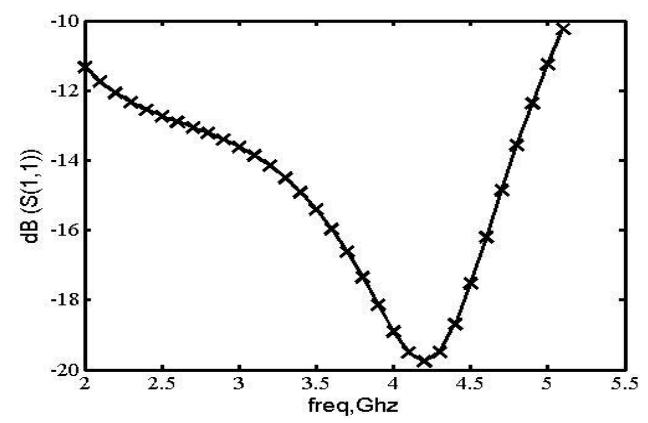

Fig. 5. Simulated S-parameters,S11

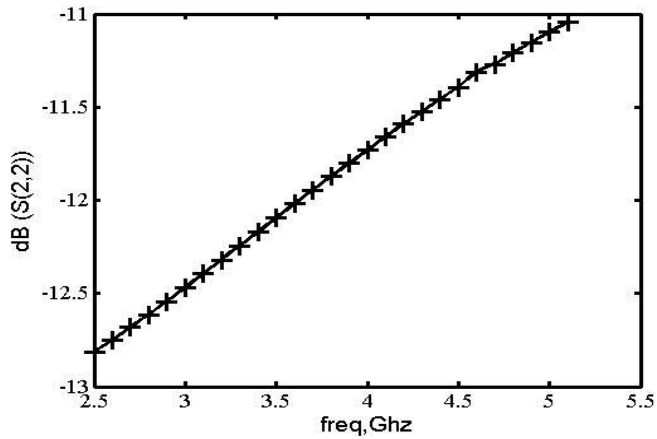

Fig. 6. Simulated S-parameters, S22

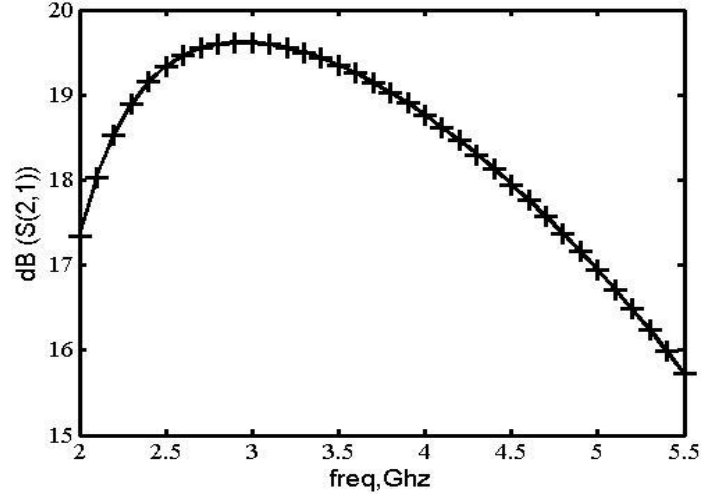

Fig. 7. Simulated S-parameters, S21

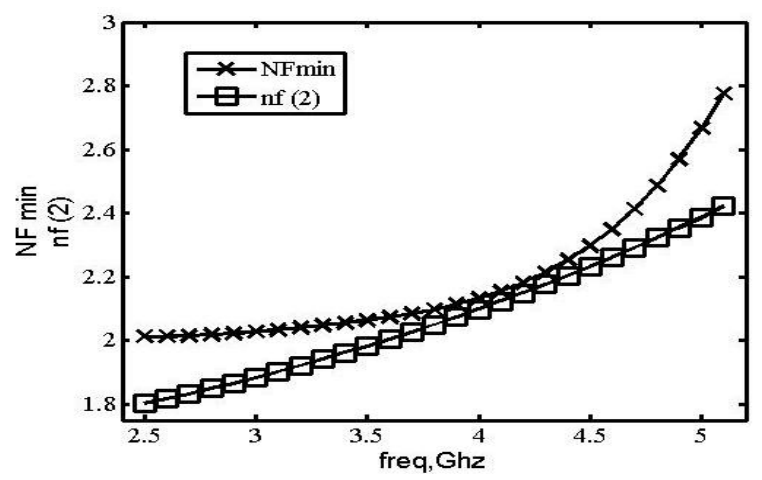

Fig. 8. Simulated NF and NFmin of the Complete LNA

\section{Conclusions}

In this paper, we design an ultra-wideband low-noise amplifier with differential output circuit based on the noise cancellation technology. Based on $0.18 \mu \mathrm{m}$ CMOS technology, the simulation is performed over $3 \mathrm{GHz}-5 \mathrm{GHz}$ bandwidth. The result shows that the voltage gain reaches $17.3 \mathrm{~dB}-19.5 \mathrm{~dB}$, noise figure is less than $2.7 \mathrm{~dB}$, and the circuit consumes $12.5 \mathrm{~mW}$ under a $1.8 \mathrm{~V}$ supply voltage. Compared with the related work, the design of low-noise amplifier achieves a better result.

Table II Comparison of the Proposed UWB LNA with Other Reported Wideband LNA

\begin{tabular}{|c|c|c|c|c|c|}
\hline & This work & Ref. [1] & Ref. [2] & Ref. [5] & Ref. [6] \\
\hline $\mathrm{S} 11 / \mathrm{dB}$ & $<-11$ & $<-10$ & $<-10$ & $<-11$ & $<-10$ \\
\hline $\mathrm{S} 21 / \mathrm{dB}$ & $>17$ & 18 & $13-15.6$ & 9.7 & $18-19.6$ \\
\hline $\mathrm{NF} / \mathrm{dB}$ & $<2.7$ & $4.5-5.5$ & $<3.5$ & $4.5-5.1$ & $<5$ \\
\hline Power/mW & 12.5 & 16 & 21 & 20 & 16.2 \\
\hline $\begin{array}{c}\text { Supply } \\
\text { Voltage/V }\end{array}$ & 1.8 & 1.2 & 1.2 & 1.8 & 1.2 \\
\hline BW/GHz & $3-5$ & $0.5-7$ & $0.2-5.2$ & $1.2-11.9$ & $2.7-4.5$ \\
\hline
\end{tabular}




\section{References}

[1] Stephan C. Blaakmeer, Eric A. M. Klumperink, Domine M. W. Leenaerts, and Bram Nauta," The BLIXER, a Wideband Balun-LNA-I/Q-Mixer Topology" in IEEE JSSC, 2008, 43(12): 2706-2714.

[2] Stephan C. Blaakmeer, Eric A. M. Klumperink, Domine M. W. Leenaerts, and Bram Nauta," Wideband Balun-LNA With Simultaneous Output Balancing, Noise-Canceling and Distortion-Canceling" in IEEE JSSC, 2008, 43(6): 341-1350.

[3] Bmccoleri F, "Wideband CMOS Low Noise Amplifier Exploiting Thermal Noise Canceling". IEEE JSSC, 2004, 39(2): 275-282.
[4] S. C. Blaakmeer, E. A. M. Klumperink, D. M. W. Leenaerts, and B.Nauta, "An inductorless wideband balun-LNA in $65 \mathrm{~nm}$ CMOS with balanced output," in Proc. 33rd Eur. Solid-State Circuits Conf. (ESSCIRC2007), Munich, Germany, Sep. 2007, pp. 364-367

[5] Chih-Fan Liao, Shen-Iuan Liu, "A Broadband Noise-Canceling CMOS LNA for 3.1-10.6GHz UWB Receivers," in IEEE JSSC, 2007, 42(2): 329-339.

[6] S. C. Blaakmeer, E. A. M. Klumperink, D. M. W. Leenaerts, and B. Nauta, "A wideband noise-canceling CMOS LNA exploiting a transformer," in 2006 IEEE RFIC Symp. Dig. Papers, Jun. 2006, pp. $137-140$. 